\title{
L'écoute : une expérience esthétique, sensible et critique de l'environnement
}

\section{Magali Babin}

\section{(2) OpenEdition \\ Journals}

Édition électronique

URL : http://journals.openedition.org/ere/2439

DOI : 10.4000/ere.2439

ISSN : 2561-2271

Éditeur

Centr'ERE

Référence électronique

Magali Babin, «L'écoute : une expérience esthétique, sensible et critique de l'environnement »,

Éducation relative à l'environnement [En ligne], Volume 14 - 1 | 2017, mis en ligne le 15 septembre 2017, consulté le 21 février 2020. URL : http://journals.openedition.org/ere/2439 ; DOI : 10.4000/ere.2439 


\title{
L'écoute : une expérience esthétique, sensible et critique de l'environnement
}

\author{
Magali Babin
}

1 En 1972, se tenait à Stockholm, la première conférence des Nations Unies sur les enjeux de la détérioration de l'environnement humain. L'éducation fut alors reconnue comme essentielle à la prévention et à la conscientisation des problèmes environnementaux. C'est ainsi que, parrainé par l'UNESCO et le Programme des Nations unies pour l'environnement, le Programme international d'éducation relative à l'environnement (PIEE) fut mis sur pied. Une année auparavant, en 1971 à Vancouver, Raymond Murray Schafer débutait un projet de recherche à l'université Simon Fraser. Le World Soundscape Project se présentait à l'époque comme la première étude mondiale sur l'environnement sonore. Compositeur, chercheur et précurseur dans ce domaine particulier qu'est la dimension sonore du monde, Schafer développa une réelle pédagogie de l'écoute de l'environnement et introduisit le concept de Paysage sonore ${ }^{1}$ qui est aujourd'hui utilisé autant dans le domaine de l'art que dans celui de la recherche sur l'écologie et les milieux naturels.

2 Je suis une artiste du son. Ma sensibilité, mon imagination et ma façon d'être au monde passent par l'écoute. Je m'exprime à travers une pratique qui donne à vivre (et à penser) des expériences sensibles utilisant la dimension sonore du monde pour favoriser un état introspectif. Depuis une dizaine d'années, les expériences et projets que je réalise m'amènent à considérer l'écoute comme une pratique qui favorise un rapport sensible à soi, à l'autre et à l'environnement. Au fil de cette pratique en création, j'ai également développé une approche pédagogique de l'écoute. À travers différents projets destinés aux jeunes publics dans les écoles primaires ou en collaboration avec des organismes communautaires, j'ai expérimenté l'écoute collective sous différentes formes : l'improvisation musicale, l'enregistrement d'environnements sonores variés, l'installation sonore comme dispositif d'exposition, etc. Toutes ces activités ont été observées et appréciées par les collaborateurs, les spectateurs et les 
participants comme des moyens efficaces pour soutenir le développement de compétences personnelles et collectives (concentration, attention, créativité) axées sur une qualité d'écoute de soi, de l'autre et de son environnement.

Cet article se penche sur les dimensions physiques et psychiques de l'espace sonore en proposant l'écoute comme une expérience esthétique et sensible du réel en relation avec les sphères environnementale, sociale et privée ${ }^{2}$. J'expliquerai en quoi l'écoute peut être envisagée dans une perspective pluridisciplinaire qui permet également d'ouvrir les consciences et d'acquérir une posture critique face aux politiques qui touchent les questions environnementales, urbanistiques et sociales.

\title{
Le son et l'espace physique
}

\author{
We must learn how to listen. It seems to be a \\ habit we have forgotten. We must sensitize the \\ ear to the miraculous world of sound around us. \\ After we have developed some critical acumen, \\ we may go on to larger projects with social \\ implications so that others may be influenced by \\ our experiences. (Schafer, 1992, p. 11)
}

L'espace physique (ou l'environnement) est l'extérieur de la psyché humaine. Il comprend l'environnement spatial et tout l'ensemble contextuel de la vie: «L'environnement n'est pas seulement l'espace qui entoure un sujet, mais aussi tout l'ensemble des conditions physiques et relationnelles dans lesquelles le sujet se trouve, agit, se définit. " (Quinz, 2004, p. 103). Le compositeur et théoricien Raymond Murray Schafer s'est intéressé à l'acoustique de notre environnement, c'est-à-dire à son écologie sonore ${ }^{3}$. À partir de 1971, il participe à un groupe de recherche sur le son au département des communications de l'Université Simon Fraser de Vancouver. Son projet mondial d'environnement sonore (World Soundscape Project) a pour but d'établir une étude comparative des paysages sonores du monde. En 1977, il publie The Tuning of the World, son maître livre qui lui vaut une reconnaissance mondiale. Précurseur dans le domaine, Murray Schafer demeure une référence pour les professionnels de domaines aussi variés que les sciences de l'environnement, l'architecture, l'urbanisme, la musique et les arts sonores. Schafer développe d'abord la notion d'événement sonore (sound-event), il s'intéresse alors à la relation intrinsèque entre la source sonore et son contexte. Selon lui, le contexte définit différents paramètres qui permettent une analyse approfondie de l'événement et, inversement, l'événement sonore fournit une panoplie d'indices « anthropologiques » du territoire écouté : «[...] l'événement sonore se définit [...] dans ses dimensions symbolique, sémantique et structurelle. Il constitue un point de référence dans le concret, lié à l'ensemble qui le dépasse » (Schafer, 1979, p. 375). Devant ce constat, Schafer expose sa théorie du paysage sonore à partir de laquelle il élabore une véritable pédagogie de l'écoute de l'environnement basée sur des expérimentations concrètes sur le terrain. Il développe ainsi une terminologie variée et précise de l'écologie sonore. 


\section{L'empreinte sonore}

5 L'empreinte sonore est définie par Schafer comme «[les] sons d'une communauté, uniques ou possédant des qualités qui les font reconnaître des membres de cette communauté ou ont pour eux un écho particulier» (2010, p. 382). L'empreinte sonore est donc un paramètre contextuel, puisqu'elle est liée à l'identité d'un territoire et des gens qui y habitent. Elle définit les particularités sonores d'un paysage, ce qui le distingue, le rend unique, surtout pour le visiteur de passage, car, bien souvent, elle constitue une habitude auditive fondue dans le quotidien de l'habitant. En ce sens, l'empreinte sonore s'inscrit dans l'identification des espaces de vie et marque la mémoire individuelle et collective des habitants de ces lieux. Le travail de Murray Schafer est des plus révélateurs sur l'importance de l'éveil de l'ouie à notre environnement, mais aussi sur nos habitudes de vie. Son approche est essentiellement axée sur une esthétique sélective, discriminant certains sons qualifiés de bruits nuisibles et menaçants pour l'écologie sonore de la nature qui foisonne de nuances riches, mais subtiles. Or, nous, humains, sommes producteurs des bruits qui meublent les milieux dans lesquels nous évoluons. Bien qu'il ne soit pas question de relancer ici le débat sur l'évolution industrielle et la pollution sonore, je retiens des recherches de R. Murray Schafer l'importance du contexte et de la source du son en relation avec la notion d'empreinte sonore.

\section{L'espace urbain ou le sonore générique}

Le bruit des grandes villes, ce murmure incessant, possède une certaine uniformité, au diapason des moteurs, même s'il se module selon les heures, parfois aux rythmes des saisons. Ce bourdonnement citadin peut-il aussi se singulariser, ou bien ses qualités acoustiques sont-elles devenues universelles? Comment, dans cette masse monotone, s'exerce la perception d'empreintes sonores? Dans Le sonore, l'imaginaire et la ville, Henri Torgue (2012, p. 133) parle de ce phénomène d'uniformité en le qualifiant d'« urbain sonore générique» parce que, selon lui, contrairement à la vue qui détaille et singularise, l'ouïe procède par référents. Henri Torgue est sociologue, urbaniste et compositeur. Il est également directeur de l'unité de recherche CNRS-MCC Ambiances architecturales et urbaines de l'École nationale supérieure d'architecture de Grenoble, et membre du Centre de recherche sur l'espace sonore et l'environnement urbain (CRESSON) de la même institution. Avec Jean-François Augoyard, fondateur du CRESSON, il a écrit À l'écoute de l'environnement. Répertoire des effets sonores (1995). Cet ouvrage est un outil singulier qui utilise l'effet sonore comme une notion servant à décrire les nuances des phénomènes acoustiques qui constituent le paysage sonore d'un lieu. Ce glossaire des qualités et des composantes sonores permet de mieux saisir leur nature et leur statut par la description détaillée de six catégories d'effets. Il est possible, par une écoute profonde et microscopique, d'entrer dans la dimension des subtilités et des nuances sonores qui nous frôlent chaque instant, et ce, même dans un milieu urbain. Être à l'écoute des empreintes sonores qui définissent un territoire, c'est aussi être à l'écoute de ses propres souvenirs et référents.

L'ouïe apparaît comme le sens qui donne accès à la part la plus intime et la plus profonde de notre être. [...] Le sonore est un facteur de captation et d'expression des émotions à la fois au plan individuel et au plan collectif. (Torgue, 2012, p. 127) 
7 L'idée à retenir ici est qu'à partir d'un " générique sonore ", il est possible de déceler par une écoute » microscopique », les détails et les particularités d'un paysage aussi dense qu'un milieu urbain et, par conséquent, d'être à l'écoute des empreintes sonores qui le définissent.

\title{
Le son et l'espace psychique
}

\author{
C'est bien dans une production de sens qu'il s'agit \\ de s'engager en écoutant, s'il est vrai « qu'être à \\ l'écoute ", c'est être disposé à l'entame du sens, \\ et donc à une entaille, à une coupure dans \\ l'indifférence in-sensée [...]. (Nancy, 2002, tel que \\ cité par Davila, 2010, p. 208)
}

\section{Le silence}

8 L'expérience vécue par John Cage en 1948 dans la chambre anéchoïque (chambre sourde) de l'Université Harvard est aujourd'hui bien connue des spécialistes du son. Elle lui révéla une vérité qui s'avéra essentielle pour la suite de son œuvre : le silence est un leurre, le monde n'est que sonore. Dans cet espace spécialisé conçu pour réaliser des tests en l'absence de tout bruit extérieur, mais aussi de toute possibilité de réverbération sonore contre les parois, Cage fut surpris par deux sons, l'un aigu, l'autre grave. L'anecdote raconte que le son aigu était celui de son système nerveux et le grave celui de sa circulation sanguine. Même dans un contexte aussi particulier, les sons du mouvement de la vie demeurent audibles en provenance de l'espace intérieur du corps. Après cette expérience, Cage composa sa célèbre pièce 4'33". Cette œuvre, écrite pour un interprète et un instrument, est complètement muette. La partition décrit trois mouvements portant la mention "TACET », qui signifie que l'interprète doit demeurer silencieux. C'est ce que David Tudor, premier interprète de 4'33", exécuta avec brio lors de la première en 1952. Le Maverick Hall, grâce à son acoustique (construit principalement en bois) et à son contexte géographique (situé aux abords d'une forêt) offrit au spectateur la présence du son dans l'instant, la musique de l'espace.

9 John Cage aimait les sonorités du monde pour ce qu'elles sont, c'est à dire des événements de la vie, la bande-son du réel; il aimait les sons et les bruits sans discrimination. En ce sens, Cage invite à vivre l'expérience de l'écoute libre et propose un espace où l'auditeur devient compositeur en raison de ses choix, de son engagement, de sa manière singulière d'entendre: c'est ce qui fait œuvre. Cage devient un " passeur », celui avec qui il est possible d'habiter le réel par l'expérience du silence qu'il propose de vivre :

Le point de départ d'un travail possible avec le son chez Cage réside dans le souci d'œuvrer à partir d'une imperceptibilité ordinaire, d'une imperceptibilité par indifférence ou par automatisation de la perception sonore, dans le but de se donner les moyens, en affinant l'écoute, en la singularisant, de nuancer la réalité acoustique du monde. (Davila, 2010, p. 211)

10 Il y a donc chez Cage un déplacement de la subjectivité, offrant une situation d'écoute totale. Toutefois, dans le cas de 4'33", ce dernier demeure dans la tradition de la mise en scène pour ce qui est de la représentation musicale. 


\section{L'écoute immersive}

Les marches ou balades sonores sont un exemple de mise en situation d'écoute de l'environnement. Elles peuvent être urbaines, collectives, et elles proposent en général une déambulation silencieuse, immersive, où chacun y va de ses observations qu'il pourra partager, ou non, selon les consignes. Dans De l'inframince. Brève histoire de l'imperceptible, de Marcel Duchamps à nos jours (2010), Thierry Davila consacre un chapitre au travail de l'artiste du son Max Neuhaus. Il se penche plus particulièrement sur deux de ses œuvres majeures pour illustrer ce qu'il nomme l'expérience du seuil chez Neuhaus. L'une de ces œuvres est Listen (1966), l'une des premières interventions dans l'espace public de l'artiste. Celle-ci consistait à une déambulation dans la ville de New York orchestrée par l'artiste qui avait préalablement tamponné sur la main de chacun des participants le mot ÉCOUTER. Cette intervention reprend l'expérience de l'écoute du «silence » de Cage en la déplaçant littéralement dans le réel, c'est-à-dire en dépassant le cadre de la représentation musicale et en confrontant le corps en mouvement au contexte de l'écoute «hors les murs» (hors de l'institution) : «Le lieu de l'art s'est déplacé et, avec lui, le corps de l'artiste, se projetant à présent volontiers dans le maillage du monde » (Ardenne, 2002, p. 89). Il s'agit d'une expérience d'écoute immersive, une marche sonore, durant laquelle l'auditeur vit une expérience physique et psychique, c'est-à-dire une situation d'écoute en mouvement avec un déplacement du corps et une spatialisation des événements sonores dans leur contexte.

Listen, avec les déambulations que cette action met en jeu, immerge spontanément le marcheur dans un univers sonore qui est l'état du monde tel qu'il existe [...] Le flâneur est donc ici amené à fréquenter et à inventer d'emblée [...]. Ces écarts remarqués, perçus et découverts, sont des seuils de perception, des seuils d'apparition et de disparition des nuances acoustiques, et c'est alors cette logique différentielle qui constitue le monde lui-même. (Davila, 2010, p. 213)

Le contexte de la ville, grouillante de bruits, est paradoxalement un terrain propice à cette introspection, à ce temps d'arrêt dans le flux de la masse sonore et anonyme. Être seul dans la foule, faire silence dans le bruit ; être écoutant, c'est éprouver l'expérience du seuil.

Du point de vue de l'immersion, ces actions témoignent d'emblée de l'ancrage
phénoménologique du travail plastique de Neuhaus, car pour le phénoménologue, il
faut que la réflexion "s'enfonce dans le monde au lieu de le dominer, qu'elle
descende vers lui tel qu'il est au lieu de remonter vers une possibilité préalable de
le penser [...], qu'elle l'interroge, qu'elle entre dans la forêt des références que notre
interrogation fait lever en lui, qu'elle lui fasse dire, enfin, ce que dans son silence il
veut dire... » (Merleau-Pondy, 1964, cité par Davila, 2010, p. 213)

Entre le silence et le dire, il y a un seuil, l'espace de la réflexion.

\section{L'expérience de l'imperceptible}

14 Par quoi cet état de connexion extérieur/intérieur (physique et psychique) peut-il être induit? Comment le son peut-il servir de vecteur entre ces dimensions? Selon Davila, les œuvres de Max Neuhaus engagent l'auditeur dans un travail de la perception qui entraîne une participation implicite :

Tel est l'engagement proprement politique de Neuhaus : mettre le visiteur face à ses responsabilités vis-à-vis du sensible, le mettre face à l'exercice de sa liberté de 
discernement, lui proposer de découvrir la manifestation d'un monde selon son bon vouloir, selon l'étendue plus ou moins affirmée de sa vigilance sensorielle. (Davila, 2010, p. 221) traduction d'une conscience éveillée. Elle devient une écoute créatrice et active dans la contemplation du moment présent. Pour mieux pénétrer et comprendre le travail de perception inscrit dans la démarche de Neuhaus, Davila s'appuie sur la phénoménologie de l'écoute de Barthes : «L'être vivant tend son audition [...] vers des indices. [...] Cette première écoute est, si l'on peut dire, une alerte.» (Barthes, tel que cité par Davila, p. 219). Barthes précise également qu'il y a une seconde écoute "qui est un déchiffrement ; ce qu'on essaie de capter par l'oreille ce sont des signes; ici sans doute, l'homme commence: j'écoute comme je lis, c'est-à-dire selon certains codes. [...] L'écoute cesse d'être pure surveillance pour devenir création.» (Barthes, tel que cité par Davila, 2010, p. 220). Les œuvres de Max Neuhaus s'immiscent dans la réalité sonore d'un contexte suscitant chez l'auditeur des moments de vigilance et de perception sur le qui-vive par un jeu de présence et d'abstraction, par «transparence ». C'est le cas de l'œuvre Times Square, une installation sonore dont le dispositif diffuse une composition électronique depuis une bouche d'aération sous les trottoirs du quartier des affaires Times Square au cœur de Manhattan. Les sonorités de cette œuvre bien connue de Max Neuhaus se "fondant dans le tissu sensationnel de la ville à l'écart inframince près. " (p. 222) L'écoute de l'auditeur est ainsi engagée dans l'attention par un jeu de distinction entre ce qu'est le paysage sonore réel et ce qu'est l'œuvre sonore.

Finalement, l'expérience du seuil, de l'imperceptible, serait un véhicule par lequel il est possible de «fracturer le réel et produire de l'écoute» (p. 220). En plongeant le visiteur dans un état de vigilance auditive, en s'immisçant dans le réel tout en créant avec, le sonore peut générer cet espace de réflexion et de connexion entre soi et l'autre, c'est-àdire le monde environnant.

\section{Conclusion}

Pour terminer, je reviendrai sur une pédagogie de l'écoute. En quoi celle-ci peut-elle favoriser la relation sensible entre l'individu et son environnement? En quoi peut-elle aider le développement d'une intégration plus active et éveillée du citoyen dans ses milieux de vie et de travail?

Dans ce sens, la pédagogie de l'écoute proposée par R. M. Schafer contribue à la (re)découverte, de notre environnement par une écoute attentive et " travaillée » selon différents exercices. Dans son livre A Sound Education (1992), Schafer développe une centaine d'exercices dédiés à l'écoute de l'environnement et à expérimenter seul ou en groupe. Il nomme ce travail de conscientisation: Ear Cleaning Exercises (Schafer, 1992). L'auteur suggère d'accompagner ces expériences par des notes personnelles et journalières en tenant un journal de bord. Personnellement, lorsque je dirige une session d'écoute ou une balade sonore, les exercices de Schafer, que je nomme des mises en situation d'écoute, sont pour moi des stratégies de transmission. Ils me permettent de communiquer ma pratique de l'écoute à travers une expérience directe et collective.

19 On ne peut parler de pédagogie de l'écoute sans mentionner également le travail et l'importante recherche qu'a menés la musicienne et compositrice Pauline Oliveros en

Éducation relative à l'environnement, Volume 14 - 1 | 2017 
élaborant la technique de l'écoute profonde, Deep Listening 4 . Dans son livre Deep Listening A Composer's Sound Practice (2005), Oliveros développe dans une perspective de transmission, cette pratique de l'écoute qu'elle a exercée toute sa vie en tant que musicienne improvisatrice, compositrice, professeure et méditante. Son approche vient d'une recherche qui joint les interrelations entre le son et l'espace; il est question d'une expérience somatique de l'écoute qu'elle soit dirigée vers une acoustique, un environnement ou une présence.

Après plusieurs années à concevoir et animer des activités pédagogiques auprès des enfants du primaire et plus spécifiquement du premier cycle, je peux affirmer que les activités interactives et expérientielles intégrant à la fois la dimension sonore, du monde et des objets, l'écoute individuelle et collective, la marche silencieuse et attentive, l'observation sonore de l'environnement, invitent l'enfant à exercer son jugement critique, à résoudre des problèmes, à développer son identité personnelle et collective, à travailler en coopération et à communiquer de façon appropriée et constructive. Les enseignants comme les parents qui ont assisté soit au déroulement de ces activités ou à leur présentation publique sont souvent surpris et touchés par la qualité de l'écoute dont font preuve les enfants lorsqu'ils sont dans l'action et la création sonore. Pour moi, ceci est révélateur: se sensibiliser à l'écoute nous rend plus concernés par ce que nous entendons. Ainsi nous pouvons imaginer que nos petits écoliers sauront être des citoyens créatifs et engagés pour participer au développement d'une réelle politique de nos espaces publics sonores. Je pense que l'écoute attentive doit faire partie des compétences à développer, et ce au plus jeune âge, puisque comme le dit si bien l'auteur de L'art comme expérience, John Dewey (2010), l'éducation est au fondement du progrès et de la réforme sociale.

\section{BIBLIOGRAPHIE}

Ardenne, P. (2002). Un art contextuel. Paris : Flammarion.

Augoyard, J.-F., Torgue, Henry. (1995). Répertoire des effets sonores. À l'écoute de l'environnement. Marseille : Éditions Parenthèses.

Babin, N. M. (2015). Prêter l'oreille : empreinte sonore et altérité : exploration de l'art en contexte dans un centre d'hébergement et de soins longue durée. (Mémoire de maîtrise). Université du Québec à Montréal. Récupéré de http://www.archipel.uqam.ca/9144/

Barthes, R. (1982). L'obvie et l'obtus essais critiques III. Paris : Éditions du Seuil.

Crocq, M. (2009) Glossaire d'écologie sonore. Journal of environmental studies, $\mathrm{n}^{\circ} 4$. Récupéré de https://www.wildproject.org/journal/4-glossaire-ecologie-sonore\#_ftn1

Davila, T. (2010). De l'inframince. Brève histoire de l'imperceptible, de Marcel Duchamps à nos jours. Paris : Regard.

Dewey, J. (2010). L'art comme expérience. Paris : Gallimard. 
Quinz, E. (2004). Espace de la présence. Notes pour une sémiologie de l'environnement sonore Dans Sobieszczanski, Marcin et Lacroix, Céline (dir.), Spatialisation en art et sciences humaines. Louvain : Éditions Peeters.

Merleau-Ponty, M. (1964). Le visible et l'invisible. Paris : Gallimard.

Nancy, J.-L. (2012). À l'écoute. Paris : Galilée.

Oliveros, P. (2005). Deep Listening. A Composer's Sound Practice. Lincoln (USA) : iUniverse, Inc.

Schafer, R. M. (1992). A Sound Education, 100 Exercises in Listening and Sound-Making. Indian River : Arcana.

Schafer, R. M. (1994). The soundscape : our sonic environment and the tuning of the world. Rochester, Verm. : Destiny Books.

Schafer, R. M. (2010). Le paysage sonore : le monde comme musique. France : Wildproject Editions.

Torgue, H. (2012). Le sonore, l'imaginaire et la ville : De la fabrique artistique aux ambiances urbaines. Paris : Éditions L' Harmattan.

\section{NOTES}

1. Schafer (2010, p. 384) définit le paysage sonore comme «l'environnement des sons. Techniquement, toute partie de cet environnement pris comme champ d'étude. Le terme s'applique aussi bien à des environnements réels qu'à des constructions abstraites, tels que compositions musicales ou montages sur bande, en particulier lorsqu'ils sont considérés comme faisant partie du cadre de vie ».

2. Le contenu de cet article est tiré et adapté de mon mémoire de maîtrise (Babin, 2015).

3. L'écologie sonore, ou écologie acoustique, est l'étude de la relation entre les organismes vivants et leur environnement sonore (Crocq, 2009).

4. Pour plus d'information sur le travail de Pauline Oliverso et la technique Deep Listening: http://deeplistening.org/site/

\section{RÉSUMÉS}

Ce texte aborde les relations entre l'être humain, l'espace sonore physique et l'espace sonore psychique. L'écoute est envisagée comme une expérience esthétique et sensible du réel dirigée vers les sphères environnementale, sociale et privée. L'espace sonore physique est caractérisé par les composantes du paysage sonore, tandis que l'espace sonore psychique répond aux qualités d'une expérience d'écoute axée sur l'attention et la vigilance. La marche sonore se présente comme une stratégie pédagogique, artistique et pluridisciplinaire visant entre autres l'adoption d'une posture critique au regard des politiques et des pratiques qui touchent les questions environnementales.

This text addresses the relationships between human, the physical sound space and the psychic sound space. Listening is seen as an aesthetic and sensitive experience of reality directed towards 
the environmental, social and private spheres. The physical sound space is characterized by the components of the soundscape, while the psychic sound space responds to the qualities of a listening experience focused on attention and alertness. The sound march is a pedagogical, artistic and multidisciplinary strategy that can invite to adopt a critical stance with regard to policies and practices that affect environmental issues.

\section{INDEX}

Mots-clés : écoute, environnement sonore, art sonore, marche sonore

Keywords : listening. sonic environment, sound art, soundwalk

\section{AUTEUR}

\section{MAGALI BABIN}

Compositrice, artiste sonore et commissaire d'évènements en art audio, Magali Babin s'est produite en concert et en performance dans le cadre de festivals internationaux au Canada, aux États-Unis, au Mexique et en Europe. Ces installations sonores ont été présentées, entre autres, au Musée d'art contemporain de Montréal (Triennale Québécoise 2011), au Mois Multi (Qc. 2012-18), à la galerie Louise et Reuben-Cohen de l'université de Moncton (2012), à la Fabrique (Nantes, France 2013) et dans le réseau des Maisons de la culture de la ville de Montréal (2015-19). En 2013, elle est boursière du Ministère de la Culture et de la Communication de France, pour une résidence de recherche à la Saline Royale (Franche-Compté). Magali Babin poursuit actuellement ses recherches dans le cadre d'un doctorat en études et pratiques des arts à l'UQAM. Elle est boursière du Fonds de recherche Société et Culture du Québec (FRQSC). 